\title{
Analytical and Computational Advances for Hydrodynamic Models of Classical and Quantum Charge Transport
}

\author{
JOSEPH W. JEROME* \\ Department of Mathematics, Northwestern University, Evanston, I1 60208
}

(Received 16 December 1998; In final form 14 December 1999)

\begin{abstract}
In recent years, substantial advances have been made in understanding hydrodynamic models, both from the standpoint of analytical infrastructure, as well as the parameters which play a decisive effect in the behavior of such models. Both classical and quantum hydrodynamic models have been studied in depth. In this survey paper, we describe several results of this type. We include, for example, well-posedness for both classical and quantum reduced models, and the relaxation drift-diffusion limit as examples of analytical results. As examples of computational results, we include some discussion of effective algorithms, but most importantly, some information gleaned from extensive simulation. In particular, we present our findings of the prominent role played by the mobilities in the classical models, and the role of hysteresis in the quantum models. All models are self-consistent. Included is discussion of recent analytical results on the use of Maxwell's equations. Benchmark devices are utilized: the MESFET transistor and the $n^{+} / n / n^{+}$diode for classical transport, and the resonant tunneling diode for quantum transport. Some comparison with the linear Boltzmann transport equation is included.
\end{abstract}

Keywords: Classical and quantum hydrodynamic models, MESFET, kinetic model, EulerMaxwell model, Riemann problems, relaxation limit

\section{INTRODUCTION}

\subsection{The Classical Hydrodynamic Model and Parameters}

The equations as presented here are discussed in references [7,27 and 22]. They are derived as the first three moments of the Boltzmann transport equation,

$$
\frac{\partial f}{\partial t}+u \cdot \nabla_{x} f-\frac{e}{m} E \cdot \nabla_{u} f=C .
$$

Here, $f=f(x, u, t)$ is the numerical distribution function of the electron species, $u$ is the species' group velocity vector, $E=E(x, t)$ is the electric field, $e$ is the electron charge modulus, $m$ is the effective electron mass, and $C$ is the time rate of change of $f$ due to collisions. The moment

*e-mail: jwj@math.nwu.edu 
equations are expressed in terms of certain dependent variables, where $n$ is the electron concentration, $v$ is the average velocity, $p$ is the momentum density, $P$ is the symmetric pressure tensor, $Q$ is the heat flux, $e_{I}$ is the internal energy, and $C_{n}, C_{p}$ and $C_{w}$ represent moments of $C$, taken with respect to the functions, $h_{0}(u) \equiv 1, h_{1}(u)=m u$, $h_{2}(u)=(m / 2)|u|^{2}$. The equations are:

$$
\begin{gathered}
\frac{\partial n}{\partial t}+\nabla \cdot(n v)=C_{n} \\
\frac{\partial p}{\partial t}+v(\nabla \cdot p)+(p \cdot \nabla) v+\nabla \cdot P=-e n E+C_{p}
\end{gathered}
$$

$$
\begin{aligned}
& \frac{\partial}{\partial t}\left(\frac{m n}{2}|v|^{2}+m n e_{I}\right)+\nabla \cdot\left(v\left\{\frac{m n}{2}|v|^{2}+m n e_{I}\right\}\right) \\
& \quad+\nabla \cdot(v P)+\nabla \cdot Q=-e n v \cdot E+C_{w} .
\end{aligned}
$$

Poisson's electrostatic equation for the electric potential must be adjoined; each species contributes a corresponding moment subsystem, with appropriately signed charge. The concentration is given by $n:=\int f d u$; the average velocity by $v:=(1 / n) \int u f d u$; the momentum by $p:=m n v$. Finally, for reference in subsequent subsections, the electron current density is given by $J:=-e n v$.

In addition to these transport equations, we have Poisson's equation for the electric field, where $n_{d}:=$ doping and $\varepsilon:=$ dielectric:

$$
\begin{aligned}
E & =-\nabla \phi, \\
\nabla \cdot(\varepsilon \nabla \phi) & =-\sum e_{i} n_{i}-n_{d} .
\end{aligned}
$$

Here, we have used the convention that there are different species, each of concentration $n_{i}$ and charge $e_{i}$. The entire system consists of Eqs. (1.2)(1.4), repeated according to species, with possible coupling terms, and (1.5), (1.6).

By moment closure is meant the selection of compatible relations among the variables, $\phi, n$, $v, P, e_{I}$ and $Q$. We begin by introducing a new variable $T$, the carrier temperature, defined by $P_{i j}=n k T \delta_{i j}$, where $k$ is Boltzmann's constant, and a scalar variable $w$, the total carrier energy. Reduction to a set of basic variables, $n, v, w$ and $\phi$, or a set equivalent to these, can be implemented. The internal energy is expressed by $m e_{I}=(3 / 2) k T$. We then have:

1. The total energy density (per unit volume) $w$ is given by combining internal energy and parabolic energy bands with $m$ assumed constant:

$$
w=m n e_{I}+\frac{1}{2} m n|v|^{2} .
$$

2. The heat flux is obtained by a differential expression involving the temperature ( $c f$. (1.9) to follow).

The final step deals with the replacement of the collision moments. For a one carrier system, we define $C_{n}=0$, and the momentum and energy relaxation times, $\tau_{p}$ and $\tau_{w}$, respectively, in terms of averaged collision moments as follows. We have:

1. The momentum relaxation time $\tau_{p}$ is given via

$$
\frac{p}{\tau_{p}}:=-m \int u C d u:=-C_{p} .
$$

2. The energy relaxation time $\tau_{w}$ is given via

$$
-\frac{w-w_{0}}{\tau_{w}}:=\frac{m}{2} \int|u|^{2} C d u:=C_{w} .
$$

Here, $w_{0}$ denotes the rest energy, $(3 / 2) k T_{0}$, where $T_{0}$ is the lattice temperature.

The forms for the relaxation times selected at $300 \mathrm{~K}$ are the Baccarani-Wordeman models [5],

$$
\begin{gathered}
\tau_{p}=\tau_{p 0}\left(\frac{T}{T_{0}}\right)^{-1}, \\
\tau_{w}=\tau_{w 0} \frac{T}{T+T_{0}}+\frac{1}{2} \tau_{p} .
\end{gathered}
$$

Here, $\quad \tau_{p 0}=(m / e) \mu_{0}$ and $\tau_{w 0}=\left(3 k T_{0} \mu_{0} / 2 e v_{s}^{2}\right)$, where $v_{s}$ is the saturation velocity. Typically, the low field mobility $\mu_{0}$ is taken to be constant, but 
other choices are possible, such as doping dependent mobilities. Units are given as $\mu \mathrm{m}^{2} / \mathrm{V} / \mathrm{ps}$.

The traditional form of $Q$ has been (see [31] for more general expressions),

$$
Q=-\kappa \nabla T \text {. }
$$

Here, $\kappa$ is the thermal conductivity governed by the Wiedemann-Franz law. This may be described by,

$$
\kappa=\frac{5 r}{2} n \frac{k^{2} \mu_{0}}{e} T\left(\frac{T}{T_{0}}\right)^{-1},
$$

where $r$ is typically taken to be .6 , giving a value of 1.5 for the dimensionless coefficient. The wellposedness of the reduced hydrodynamic model, for two carriers, was demonstrated in [11].

\subsection{The Drift-Diffusion Model}

The drift-diffusion model may be obtained by taking zeroth order moments of the BTE and adjoining the Poisson equation. Thus, one obtains the system for $N$ carriers with recombination $R_{i}$, current density $J_{i}$, (signed) charge $e_{i}, i=1, \ldots, N$ :

$$
\frac{e_{i} \partial n_{i}}{\partial t}+\nabla \cdot J_{i}=-e_{i} R_{i}
$$

together with (1.5) and (1.6). There still remains the issue of determining the constitutive current relations. Classical drift-diffusion theory gives, for $N=2, n_{1}=n$ and $n_{2}=p$,

$$
\begin{aligned}
& J_{n}=-e \mu_{n} n \nabla \phi+e D_{n} \nabla n, \\
& J_{p}=-e \mu_{p} p \nabla \phi-e D_{p} \nabla p .
\end{aligned}
$$

The electronic charge modulus $e$ is positive here, and $n$ and $p$ denote the electron and hole densities, respectively. The use of the Einstein relations linking the mobilities, $\mu_{n}, \mu_{p}$, and the diffusion coefficients, $D_{n}, D_{p}$, is common. These relations are specified by

$$
D_{n}=(k T / e) \mu_{n},
$$

$$
D_{p}=(k T / e) \mu_{p}
$$

A detailed existence and approximation theory for this model was presented in [22].

\subsection{The Quantum Hydrodynamic Model}

The model used in this paper was derived by Gardner in [20]. In this section, we shall review the basic characteristics of the model as described in [14]. An existence theorem for the reduced model was obtained in [33]. The model is also discussed in [22].

The QHD model has exactly the same structure as the classical hydrodynamic model (electrogasdynamics), where we now permit a non-isotropic stress tensor:

$$
\begin{gathered}
\frac{\partial n}{\partial t}+\frac{\partial}{\partial x_{i}}\left(n v_{i}\right)=0 \\
\frac{\partial}{\partial t}\left(m n v_{j}\right)+\frac{\partial}{\partial x_{i}}\left(v_{i} m n v_{j}-P_{i j}\right)=-n \frac{\partial V}{\partial x_{j}}-\frac{m n v_{j}}{\tau_{p}} \\
\frac{\partial w}{\partial t}+\frac{\partial}{\partial x_{i}}\left(v_{i} w-v_{j} P_{i j}+q_{i}\right) \\
=-n v_{i} \frac{\partial V}{\partial x_{i}}-\frac{\left(w-(3 / 2) n T_{0}\right)}{\tau_{w}}
\end{gathered}
$$

in conjunction with Poisson's equation, (1.6), where $V=-e \phi$ is the potential energy, and temperature here is expressed in energy units $(k$ is set equal to 1). Spatial indices $i, j$ equal $1,2,3$, and repeated indices are summed over.

Quantum mechanical effects appear in the stress tensor and the energy density. Gardner derived the stress tensor and the energy density based upon the $O\left(\hbar^{2}\right)$ momentum-shifted thermal equilibrium Wigner distribution function:

$$
\begin{aligned}
P_{i j} & =-n T \delta_{i j}+\frac{h^{2} n}{12 m} \frac{\partial^{2}}{\partial x_{i} \partial x_{j}} \log (n)+O\left(h^{4}\right), \\
w & =\frac{3}{2} n T+\frac{1}{2} m n u^{2}-\frac{\hbar^{2} n}{24 m} \nabla^{2} \log (n)+O\left(\hbar^{4}\right) .
\end{aligned}
$$


In one dimension, the QHD model requires eight boundary conditions. Well-posed boundary conditions for the resonant tunneling diode are $n=n_{d}, \partial n / \partial x=0$, and $\partial T / \partial x=0$ at the left and right diode boundaries $x_{L}$ and $x_{R}$, with a bias $\Delta V$ across the device: $V\left(x_{L}\right)=T \log \left(n / n_{*}\right)$ and $V\left(x_{R}\right)=T \log \left(n / n_{*}\right)+e \Delta V$, where $n_{*}$ is the intrinsic electron concentration.

\subsection{Euler-Maxwell Systems}

We shall also examine a form of the EulerMaxwell system for semiconductor transport. In particular, we shall consider the 'isentropic' mass-momentum transport system, coupled to Maxwell's equations for the electric and magnetic fields, instead of Poisson's equation for the electric field only. This Euler-Maxwell system in the isentropic case assumes the following form $[3,4]$ :

$$
\left\{\begin{array}{l}
n_{t}+\nabla \cdot(n v)=0, \\
(n v)_{t}+\nabla \cdot(n v \otimes v)+\nabla p(n) \\
\quad=-\frac{e}{m} n(E+v \times B)-\frac{n v}{\tau}, \\
\mu H_{t}+\nabla \times E=0, \\
\varepsilon E_{t}-\nabla \times H+J=0, \\
-\varepsilon \nabla \cdot E=e n-n_{d}(x), \quad \nabla \cdot H=0, \\
B=\mu H, \quad J=-e n v, \quad x \in \mathbf{R}^{3}, \quad t>0,
\end{array}\right.
$$

where $p=p(n)=n^{\gamma} / \gamma$ is the pressure of the flow, expressed in normalized units, $\gamma>1$ is the adiabatic exponent, $H \in \mathbf{R}^{3}$ is the magnetic field, $B \in \mathbf{R}^{3}$ is the magnetic induction, $-e(E+v \times B)$ is the Lorentz force, and $\mu$ is the permeability of the medium.

\section{RELAXATION LIMIT TO DRIFT - DIFFUSION EQUATIONS}

In this section we study the singular limit of (1.2)(1.6) when the relaxation times $\tau_{w}$ and $\tau_{p}$ tend to zero with $0 \leq 2 \tau_{w}-\tau_{p} \leq M \sqrt{\tau_{p} \tau_{w}}$. An examination of the relaxation expressions $(1.7,1.8)$ reveals that this is equivalent to the following parametric limit:

$$
\text { - } \frac{\mu_{0}}{v_{s}} \rightarrow 0, \quad \text { with } v_{s} \geq v_{*}>0 .
$$

We first scale the variables $(n, v, T, \phi)$, and then show that the limit functions satisfy the drift-diffusion system as $\tau_{p}, \tau_{w} \rightarrow 0$ in the manner specified.

Let

$$
\begin{aligned}
& n^{\tau}(x, s)=n\left(x, \frac{s}{\tau_{p}}\right), \quad v^{\tau}(x, s)=\frac{1}{\tau_{p}} v\left(x, \frac{s}{\tau_{p}}\right), \\
& \theta^{\tau}(x, s)=\frac{1}{\tau_{w}}\left(T\left(x, \frac{s}{\tau_{w}}\right)-\bar{T}\right), \\
& \phi^{\tau}(x, s)=\phi\left(x, \frac{s}{\tau_{p}}\right), \quad n_{d}^{\tau}(x, s)=d\left(x, \frac{s}{\tau_{p}}\right) .
\end{aligned}
$$

Then (1.2)-(1.6) is transformed as follows, if $\tau_{p}, \tau_{w}, \kappa$ are treated as constants:

$$
n_{s}^{\tau}+\left(n^{\tau} v^{\tau}\right)_{x}=0
$$

$$
\tau_{p}^{2} v_{s}^{\tau}+\tau_{p}^{2} v^{\tau} v_{x}^{\tau}+\frac{1}{n^{\tau}}\left(\left(\tau_{w} \theta^{\tau}+\bar{T}\right) n^{\tau}\right)_{x}+v^{\tau}=\phi_{x}^{\tau},
$$

$$
\begin{gathered}
\tau_{w}^{2} \theta_{s}^{\tau}-\frac{2 \kappa \tau_{w}}{3 n^{\tau}} \theta_{x x}^{\tau}+\tau_{p} \tau_{w} v^{\tau} \theta_{x}^{\tau}+\frac{2 \tau_{p}}{3}\left(\tau_{w} \theta^{\tau}+\bar{T}\right) v_{x}^{\tau} \\
-\frac{\tau_{p}\left(2 \tau_{w}-\tau_{p}\right)}{3 \tau_{w}}\left(v^{\tau}\right)^{2}+\theta^{\tau}=0 \\
\phi_{x x}^{\tau}=n^{\tau}-n_{d}^{\tau}
\end{gathered}
$$

with the following initial-boundary data on the interval $(0,1)$ :

$$
\begin{aligned}
& \left(n^{\tau}, v^{\tau}, \theta^{\tau}\right)=\left(n_{0}(x), v_{0}(x), T_{0}(x)-\bar{T}\right), \\
& \left(v^{\tau}, \theta_{x}^{\tau}, \phi^{\tau}\right)(i, s)=0, \quad i=0,1, \quad s \geq 0 .
\end{aligned}
$$

We assume that initial data $\left(n_{0}(x), v_{0}(x)\right.$, $\left.T_{0}(x)-\bar{T}\right)$ are independent of $\tau_{p}$ and $\tau_{w}$ and are "small" as measured in appropriate norms.

Theorem 2.1 Assume that a unique smooth solution, $(n, v, T, \phi)$, exists for (1.2)-(1.6). Then there exists $\nu(x, s)$ such that

$$
\begin{gathered}
\left(n^{\tau}, \phi_{x}^{\tau}\right) \rightarrow\left(\nu, \int_{0}^{1} \int_{\eta}^{x}\left(\nu(\xi, s)-n_{d}(\xi)\right) d \xi d \eta\right) \\
\text { a.e. as } \tau_{p}, \tau_{w} \rightarrow 0 .
\end{gathered}
$$


The limit function $\nu$ satisfies the drift-diffusion equation,

$$
\nu_{s}+\left(\nu \int_{0}^{1} \int_{\eta}^{x}\left(\nu(\xi, s)-n_{d}(\xi)\right) d \xi d \eta-\nu_{x}\right)_{x}=0
$$

in the sense of distributions. The double integral may be identified with $\Phi_{x}$, where $\Phi$ is the electrostatic potential in the Poisson equation. Note that physical constants have been homogenized to unity in the limiting equation.

\section{RESULTS ON WELL-POSEDNESS FOR THE CLASSICAL MODEL}

A detailed study of the well-posedness of the initial/boundary-value problem for the reduced hydrodynamic model was carried out in [11]. The reduction refers to the assumption of a pressure-density relationship of adiabatic type, and the subsequent elimination of the energy equation from the system. The mathematical theory of (perturbed) conservation laws is not capable of handling the full system in terms of demonstrating the existence of physical solutions to the initial/ boundary-value problem. We briefly sketch a few rudiments of the theory in the following subsections. The interested reader may consult the references for elaboration. The basic idea is to construct approximate solutions, via so-called Riemann problems. The extraction of a convergent subsequential limit involves a relatively new idea of compensated compactness. The analysis of [11] actually includes the two-carrier case, as well as more complicated equations arising from multidimensional symmetry reduction to one dimension. We now describe the basic idea of Riemann problems in the homogeneous case.

\subsection{Riemann Problems as Building Blocks}

In this section, we review some basic facts about the Riemann solutions for homogeneous systems. Although the reduced hydrodynamic model represents a perturbation of such a system, the homogeneous system is the starting point in the construction of the approximate solutions. We find it convenient to use the notation $\rho=m n$, and formulate the system in terms of $\rho$, the mass density. Consider the homogeneous system:

$$
u_{t}+f(u)_{x}=0, \quad 0<x<1,
$$

where $u=(\rho, p)^{\top}$ and $f(u)=\left(p,\left(p^{2} / \rho\right)+P(\rho)\right)^{\top}$ with $P(\rho)=\rho^{\gamma} / \gamma, \gamma>1$.

The discontinuity in the weak solution of (3.1) satisfies the Rankine-Hugoniot condition:

$$
\sigma\left(u-u_{0}\right)=f(u)-f\left(u_{0}\right),
$$

where $\sigma$ is the propagation speed of the discontinuity, and $u_{0}$ and $u$ are the corresponding left state and right state, respectively. The shock with speed $\sigma=0$ is called the standing shock.

Consider the Riemann problem consisting of (3.1) with initial data,

$$
\left.u\right|_{t=0}= \begin{cases}u_{-}, & x<x_{0}, \\ u_{+}, & x>x_{0},\end{cases}
$$

where $x_{0} \in(0,1), u_{ \pm}=\left(\rho_{ \pm}, p_{ \pm}\right)^{\top}$, and $\rho_{ \pm} \geq 0$ and $p_{ \pm}$ are constants satisfying $\left|\left(p_{ \pm} / \rho_{ \pm}\right)\right|<\infty$.

For the Riemann problem with data (3.3) and the Riemann initial-boundary problem of (3.1) with data:

$$
\left.u\right|_{t=0}=u_{+},\left.\quad p\right|_{x=0}=0
$$

we have the following facts regarding the solutions.

Lemma 3.1 There exists a piecewise smooth entropy solution $u(x, t)$ for each of the problems (3.3) and (3.4), respectively, satisfying an invariant region condition for some region $\sum$. That is, if the Riemann data lie in $\sum$, then the Riemann solutions $u(x, t) \in \sum$ and $(1 /(b-a)) \int_{a}^{b} u(x, t) d x \in \sum$. For the Riemann initial-boundary problem of (3.1) with data:

$$
\left.u\right|_{t=0}=u_{-},\left.\quad p\right|_{x=1}=0
$$


we have the similar results to those for (3.4). The entropy solutions are the physically relevant solutions.

The method by which the Riemann solutions are utilized in the reduced hydrodynamic model is by a variant of Godunov's method, well-known in gas dynamics, where typically there are no forcing terms. Piecewise constant starting values, an approximation to initial data, are used to construct solutions locally in time by the theory of Riemann solvers; the perturbation terms are evaluated explicitly via a fractional step, and the procedure is repeated to advance in time. The Courant-Friedrich's condition on the time step is essential for the construction. One thereby obtains a family of approximate solutions, indexed by spatial grid length. The next subsection sketches the underlying theory which permits the extraction of a limit.

\section{2. $H^{-1}$ Compactness of Entropy Measures}

In the theory of conservation laws, the notion of entropy pairs, or weak entropy pairs, plays a decisive role. They are (usually) convex functions which may be thought of as facilitating the estimates. We have the following. A pair of mappings $(\eta, q): \mathbf{R}^{2} \rightarrow \mathbf{R}^{2}$ is called an entropy-entropy flux pair if $\nabla q=\nabla \eta \nabla f$. If $\tilde{\eta}(\rho, v) \equiv \eta(\rho, \rho v)$ satisfies $\tilde{\eta}(0, v)=0$, for any fixed $v=(p / \rho)$, then $\eta$ is called a weak entropy. For example, the mechanical energy-energy flux pair

$$
\begin{aligned}
& \eta_{*}=\frac{1}{2} \frac{p^{2}}{\rho}+\frac{1}{\gamma(\gamma-1)} \rho^{\gamma}, \\
& q_{*}=p\left(\frac{1}{2} \frac{p^{2}}{\rho^{2}}+\frac{\rho^{\gamma-1}}{\gamma-1}\right),
\end{aligned}
$$

is a strictly convex weak entropy pair for (3.1).

In order to extract convergent sequences from approximate solutions, it is necessary to have a compactness criterion in function space. We need the following basic lemma ( $c f$. [10,16,32]). For readers not familiar with the notation of the following lemma, we are employing negatively indexed (dual) Sobolev spaces of distributions.

Lemma 3.2 Let $\Omega \subset \boldsymbol{R}^{N}$ be a bounded domain. Then

$$
\begin{aligned}
& \text { (compact set of } \left.W^{-1, q}(\Omega)\right) \\
& \cap\left(\text { bounded set of } W^{-1, r}(\Omega)\right) \\
& \subset\left(\text { compact set of } W_{\text {loc }}^{-1,2}(\Omega)\right)
\end{aligned}
$$

where $q$ and $r$ are constants, $1<q \leq 2<r<\infty$.

The approximate solutions satisfy this lemma. This is expressed in the following proposition.

Proposition 3.1 If $\left\{u_{i}^{h}\right\}, i=1,2$, are the approximate solutions, then the measure sequence

$$
\eta\left(u_{i}^{h}\right)_{t}+q\left(u_{i}^{h}\right)_{x}
$$

is a compact subset of $H_{l o c}^{-1}(\Omega)$ for all weak entropy pairs $(\eta, q)$, where $\Omega$ is any bounded and open set in the space-time domain $\Pi_{T}$.

The compensated compactness framework is designed to handle the case when the approximate solutions satisfy an invariant region principle, and when the entropy pairs are compact in a suitable topology. We have the following framework (see [10]):

LEMMA 3.3 Assume that the approximate solutions $u^{h}=\left(\rho^{h}, p^{h}\right)$ satisfy

(1) There is a constant $C>0$ such that $0 \leq \rho^{h}$ $(x, t) \leq C,\left|p^{h}(x, t) / \rho^{h}(x, t)\right| \leq C$.

(2) The measure $\eta\left(u^{h}\right)_{t}+q\left(u^{h}\right)_{x}$ is compact in $H_{l o c}^{-1}(\Omega)$, for all weak entropy pairs $(\eta, q)$, where $\Omega \subset \Pi_{T}$ is any bounded and open set.

Then, for $1<\gamma \leq 5 / 3$, there exists a convergent subsequence (still labeled $u^{h}$ ) such that $u^{h}(x, t) \rightarrow$ $u(x, t)=(\rho(x, t), p(x, t))$, a.e.

This is the final component required to demonstrate that $u$ is a weak solution of the reduced hydrodynamic system. Additional references, such as [26], may be found in [11]. 


\section{EXISTENCE FOR THE QHD MODEL}

The quantum hydrodynamic (QHD) model is a moment model, derived from the Wigner equation. It may be viewed as a quantum corrected version of the classical hydrodynamic equations, with the stress tensor and the energy density corrected by $O\left(\hbar^{2}\right)$ perturbations. Some applications, such as the resonant tunnel diode, also involve quantum well potentials. Ancona, Iafrate and Tiersten $[2,1]$ derived the expression for the stress tensor, and Grubin and Kreskovsky formulated a one dimensional version of the model [21]. The model which we study is of physical importance; in fact, a simplification of our model has been characterized as a pure state, single carrier transport model in [21]. Note that a pure state model would have the factor $(-1 / 4)$ in $(4.5)$ whereas the mixed state model has the factor $(-1 / 12)$.

Our approach is completely novel to this application area, in that we reduce the system to an integro-differential equation, with a set of boundary conditions, including a nonstandard secondorder boundary condition, which is equivalent to specifying the quantum potential at the (current) inflow boundary.

\subsection{Formulation and Summary of Result}

We shall present the equations for the simplified QHD model as developed in [21] and [20].

$$
\begin{gathered}
n_{t}+(n v)_{x}=0 \\
(m n v)_{t}+\left(m n v^{2}+p(n)+Q(n)\right)_{x}=n \phi_{x}-\frac{m n v}{\tau} \\
\phi_{x x}=-e\left(n_{d}-n\right) .
\end{gathered}
$$

The pressure function, $p=p(n)$, has the property that $n^{2} p^{\prime}(n)$ is strictly monotonically increasing from $[0, \infty)$ onto $[0, \infty)$. A commonly-used hypothesis is:

$$
p(n)=k n^{\gamma}, \quad \gamma>1, k>0 .
$$

Quantum mechanics is represented by the quantum potential [1]:

$$
Q(n)=-\frac{\hbar^{2}}{12 m} n(\log (n))_{x x} .
$$

The device domain is the $x$-interval, $I \equiv(0,1)$.

In this section we describe the steady-state case $n_{t}=(n v)_{t}=0$. Then, after the introduction of the current density $j=n v$, the system (4.1)-(4.3) reduces to

$$
\begin{gathered}
j(x)=\text { const. } \\
\left(\frac{m j^{2}}{n}+p(n)+Q(n)\right)_{x}=n \phi_{x}-\frac{m j}{\tau}, \\
\phi_{x x}=-e\left(n_{d}-n\right) .
\end{gathered}
$$

Assume that $j$ is a specified positive constant. Since (4.7) is a third order ordinary differential equation, and (4.8) is Poisson's equation, three boundary conditions for (4.7) and two boundary conditions for (4.8) are prescribed as follows:

$$
\begin{gathered}
n(0)=n_{0}, \quad n(1)=n_{1}, \quad n_{0} n_{x x}(0)-\frac{1}{2} n_{x}^{2}(0)=n_{2}, \\
\phi(0)=\phi_{0}, \quad \phi(1)=\phi_{1},
\end{gathered}
$$

where $n_{0}, n_{1}$ and $n_{2}$ are positive constants; $\phi_{0}$ and $\phi_{1}$ are the applied bias potentials.

\subsection{The Integral Equation}

In order to transform the above equations, we first use a Green's function to solve Poisson's equation (4.8), and then we reduce the system (4.7) - (4.8) to an integro-differential equation. We shall see that the existence of a smooth solution of the original system is equivalent to that of a smooth solution of the integro-differential equation.

The solution of (4.8) with boundary data (4.10) is given uniquely by

$$
\phi=-e \int_{0}^{1} G(x, \xi)\left(n_{d}-n\right) d \xi+x\left(\phi_{1}-\phi_{0}\right)+\phi_{0},
$$


where $G(x, \xi)$ is the Green's function for this problem, and is defined by

$$
G(x, \xi)= \begin{cases}x(\xi-1), & x<\xi \\ \xi(x-1), & x>\xi\end{cases}
$$

We now transform the Eq. (4.7) to a secondorder ordinary differential equation by integration. Dividing (4.7) by $n$, we have

$$
-\frac{1}{n}(Q(n))_{x}=\left(\frac{m j^{2}}{2 n^{2}}+\frac{k \gamma}{\gamma-1} n^{\gamma-1}-\phi\right)_{x}+\frac{m j}{n \tau} .
$$

Since

$$
-\frac{1}{n}(Q(n))_{x}=\frac{\hbar^{2}}{6 m}\left(\frac{1}{\sqrt{n}}(\sqrt{n})_{x x}\right)_{x}
$$

then (4.13) becomes

$$
\begin{aligned}
& \frac{\hbar^{2}}{6 m}\left(\frac{1}{\sqrt{n}}(\sqrt{n})_{x x}\right)_{x} \\
& \quad=\left(\frac{m j^{2}}{2 n^{2}}+\frac{k \gamma}{\gamma-1} n^{\gamma-1}-\phi\right)_{x}+\frac{m j}{n \tau}
\end{aligned}
$$

Integrating (4.14) from 0 to $x$ and using the boundary data (4.9), we have

$$
\begin{aligned}
\frac{\hbar^{2}}{6 m}\left(\frac{1}{\sqrt{n}}(\sqrt{n})_{x x}\right)= & \frac{m j^{2}}{2 n^{2}}+\frac{k \gamma}{\gamma-1} n^{\gamma-1} \\
& +m j \int_{0}^{x} \frac{d y}{n \tau}-\phi \\
& +\frac{\hbar^{2}}{12 m} \frac{n_{2}}{n_{0}^{2}}-\frac{m j^{2}}{2 n_{0}^{2}} \\
& -\frac{k \gamma}{\gamma-1} n_{0}^{\gamma-1}+\phi_{0} .
\end{aligned}
$$

Let $w=\sqrt{n}$. By substituting (4.11) for $\phi$ in (4.15), the system of Eqs. (4.6)-(4.8) reduces to an integro-differential equation with Dirichlet conditions:

$$
\begin{gathered}
\frac{\hbar^{2}}{6 m} w_{x x}=\frac{m j^{2}}{2 w^{3}}+\frac{k \gamma}{\gamma-1} w^{2 \gamma-1}+m j w \int_{0}^{x} \frac{d y}{w^{2} \tau} \\
+w\left(e \int_{0}^{1} G(x, \xi)\left(n_{d}-w^{2}\right) d \xi\right. \\
\left.-x\left(\phi_{1}-\phi_{0}\right)+b\right), \\
w(0)=w_{0}, \quad w(1)=w_{1},
\end{gathered}
$$

where

$$
\begin{aligned}
b & =\frac{\hbar^{2}}{12 m} \frac{n_{2}}{n_{0}^{2}}-\frac{m j^{2}}{2 n_{0}^{2}}-\frac{k \gamma}{\gamma-1} n_{0}^{\gamma-1}, \\
w_{0} & =\sqrt{n_{0}}, \quad w_{1}=\sqrt{n_{1}} .
\end{aligned}
$$

If $w$ is a smooth solution of (4.16), (4.17), then

$$
2 w_{0}^{3} w_{x x}(0)=n_{2}
$$

That is, the third boundary condition of (4.9) holds for $w$. Hence, the existence of a smooth solution of $(4.6)-(4.10)$ is equivalent to that of a smooth solution of (4.16), (4.17), provided $n$ does not vanish on $I$. The main result was proven in [33]. With minor changes, it reads as follows (due to the correction of some sign errors in [33]):

THEOREM 4.1 Assume that

$$
\begin{aligned}
\phi_{0} & \geq \phi_{1}+\frac{e}{2}\left\|N_{D}-N_{A}\right\|_{L^{\infty}}, \\
n_{2} & \geq \frac{12 m}{\hbar^{2}}\left(\frac{m j^{2}}{2}+\frac{k \gamma}{\gamma-1} n_{0}^{\gamma+1}\right), \\
\left\|\frac{\partial \tau}{\partial n}\right\|_{L^{\infty}} & \leq M .
\end{aligned}
$$

Then, for each $j>0$, there exists a classical solution $(n, \phi)$ of (4.7)-(4.10), satisfying the properties that $n \in C_{B}^{3}(I)$ (for compact subsets of $I$ ) and $(1 / K) \leq \sqrt{n} \leq K$ for some $K>0$. 


\section{EULER-MAXWELL WELL-POSEDNESS}

We shall be brief in this section since we have already described the general approach to EulerPoisson systems in $\S 3$. The approach of [12] adopts this method, but extends the ideas to the EulerMaxwell system in one spatial variable. The critical boundary conditions, as before, are with the momentum:

$$
\left.p\right|_{x=0}=\left.p\right|_{x=1}=0 .
$$

For general large initial data in $L^{\infty}$, the solutions of (1.21) will develop singularities or shocks in finite time. Therefore, there are only global weak solutions, including shock waves, for general large initial data. The global solution of (1.21) is constructed under the assumption of a linearized Lorentz force. In this sense, the well-posedness result is partial. If the initial-boundary conditions are bounded, the global solution will be bounded. The global approximate solutions constructed by the Godunov method with the fractional step procedure, are then shown to be convergent to the global weak solution.

\section{NUMERICAL ALGORITHMS}

The simulations contained in [23, 24 and 25], as well as the earlier simulations in $[18,17]$, were based upon ENO schemes, or upon careful calibration between ENO and upwinding. Such schemes are actually discrete Godunov schemes. Alternative algorithms, particularly those based upon upwinding finite element methods, are perhaps less known, and we mention these briefly now. These were particularly successful in the studies carried out for the QHD model in [14].

The method is described as follows. First, we triangulate our domain $\Omega$ with triangulations $\mathcal{T}_{h}$ made solely of rectangles $R$ such that the intersection of two distinct rectangles of the triangulation $\mathcal{T}_{h}$ is either an edge, a vertex, or void. Then, for each $t \in\left(0, t_{f}\right]$, we take each of the components of our approximate solution $\mathbf{u}_{h}(t)$ in the space

$$
V_{h}=\left\{p \in L^{\infty}(\Omega):\left.p\right|_{R} \text { is linear, } \forall R \in \mathcal{T}_{h}\right\} .
$$

We define each of the components of $\mathbf{u}_{0 h}$ to be the $\mathrm{L}^{2}$-projection of the corresponding component of $\mathbf{u}_{0}$ into $V_{h}$ and discretize the Eq. (3.1) in space by using the Discontinuous Galerkin (DG) method. Since the functions of the space $V_{h}$ are discontinuous, the mass matrix of the DG method is block-diagonal. Thus, the resulting discrete equations can be rewritten as the following ODE initial value problem:

$$
\begin{gathered}
\frac{d \mathbf{u}_{h}}{d t}=\mathbf{L}_{h}\left(\mathbf{u}_{h}, \mathbf{g}\right)+\mathbf{R}_{h}\left(\mathbf{u}_{h}\right), \quad t \in(0, T], \\
\mathbf{u}_{h}(t=0)=\mathbf{u}_{0 h},
\end{gathered}
$$

where $\mathbf{L}_{h}$ is the approximation of $-\nabla \cdot \mathbf{F}$. The exact solution of the above initial value problem gives an approximation which is formally secondorder accurate in space; see [15]. Accordingly, a second-order accurate in time Runge-Kutta method must be used to discretize our ODE; see [15, 29 and 30]. Finally, a local projection $\Lambda \Pi_{h}$ is applied to the intermediate values of the Runge-Kutta discretization in order to enforce nonlinear stability.

The general definition of the DG method in the case of a scalar $\mathbf{u}$ can be found in [15]. To define the method in our case, we simply have to apply the procedure for the scalar case component by component. When the formal integration by parts is carried out after the equations are multiplied by test functions, the flux appears as a term of the boundary line integrals. The upwinding is applied in the definition of the flux representation. A local Lax-Friedrichs flux is typically employed.

\section{SIMULATION OF THE MESFET}

A benchmark MESFET is displayed in Figure 1. We present some recent results of our simulations, particularly with respect to symmetry and symmetry-breaking (see [11]). 


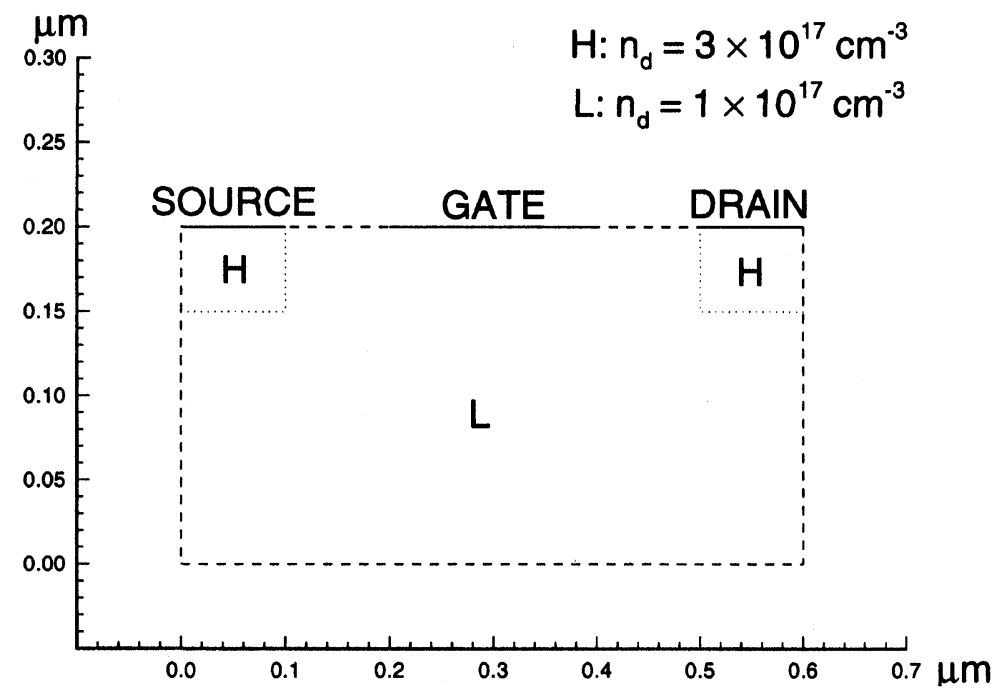

FIGURE 1 The MESFET: Geometry and Doping.
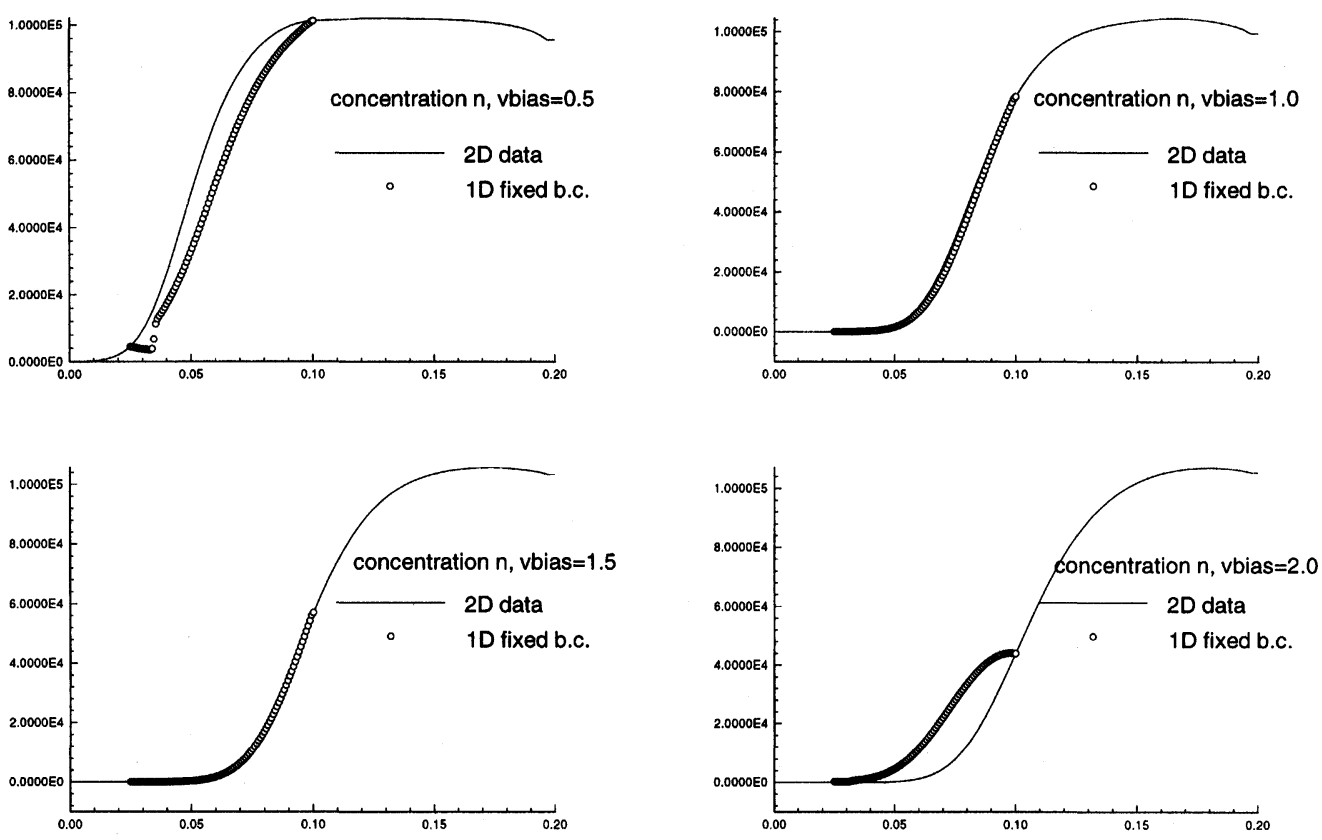

FIGURE 2 The 1D model with spherical symmetry assumption, in comparison with the 2D MESFET results, at $v$ bias $=0.5 \mathrm{~V}$, $1.0 \mathrm{~V}, 1.5 \mathrm{~V}$ and $2.0 \mathrm{~V}$. The concentration $n$.

\subsection{Symmetry Breaking Role of Bias Potentials}

Next, we show the result of using the 1D model with a spherical symmetry assumption, to approximate the 2D MESFET shown above. We take our 1D domain from $r=0.025$ to $r=0.1$, measured from the top middle point at $(x, y)=$ $(0.3,0.2)$ downward. The boundary conditions for the concentration $n$, the temperature $T$ and the potential $\phi$ are prescribed, using the values of the 2D simulations; the boundary condition for the velocity is floating (Neumann). In Figure 2, 
we show the comparison, for the concentration $n$, of the 2D MESFET result with the 1D model assuming spherical symmetry, at $v$ bias $=$ $0.5 \mathrm{~V}, 1.0 \mathrm{~V}, 1.5 \mathrm{~V}$ and $2.0 \mathrm{~V}$. We can clearly see a qualitatively correct agreement. This is very promising since it means that other quantities (such as $T$ and $\phi$ ) which are not spherically symmetric have minimal effect on the concentration through the nonlinear coupling of the equations.

\section{MOBILITY CALIBRATIONS}

This work describes results presented in [9], but not previously published. We demonstrate, by comparison of the kinetic and hydrodynamic model, the critical role played by the mobility functions. The device we consider is the one dimensional GaAs $n^{+}-n-n^{+}$structure of length $0.8 \mu \mathrm{m}$. The device used is as follows: $x \in[0,0.8]$; the doping is defined by $n_{d}(x)=10^{6} / \mu \mathrm{m}^{3}$ in $0 \leq x \leq 0.175$ and in $0.625 \leq x \leq 0.8$, and by $n_{d}(x)=2 \times 10^{3} / \mu \mathrm{m}^{3}$ in $0.225 \leq x \leq 0.575$, with a smooth intermediate transition. This is exactly the device used in Baranger and Wilkins [6], except for a smooth transition of width $0.05 \mu \mathrm{m}$ at the junctions.

We rewrite the kinetic model for the linearized version we consider. The one-dimensional kinetic model can be written as follows:

$$
\begin{aligned}
& \frac{\partial f(x, u, t)}{\partial t}+u \frac{\partial f(x, u, t)}{\partial x}-\frac{e}{m} E(x, t) \frac{\partial f(x, u, t)}{\partial u} \\
& =\frac{n(x, t) M(u)-f(x, u, t)}{\tau}
\end{aligned}
$$

where

$$
M(u)=\frac{1}{\sqrt{2 \pi \theta}} \mathrm{e}^{-u^{2} / 2 \theta}
$$

is a Maxwellian, with

$$
\theta=\frac{k_{b}}{m} T_{0}
$$

The concentration $n(x, t)$ is obtained by

$$
n(x, t)=\int_{-\infty}^{\infty} f(x, u, t) d u .
$$

Also, the electric field $E(x, t)$ is obtained by solving the coupled potential equation,

$$
E(x, t)=-\phi_{x}, \quad\left(\varepsilon \phi_{x}\right)_{x}=e\left(n-n_{d}\right),
$$

with the boundary conditions

$$
\phi(0, t)=0, \quad \phi(0.8, t)=v \text { bias },
$$

and the relaxation parameter $\tau$ is computed by

$$
\tau=\frac{m \mu}{e} .
$$

$\mu$ is the mobility and we have the following characterizations.

1. Constant $\mu$. We have used values:

$$
\begin{gathered}
\mu=0.75 \mu \mathrm{m}^{2} /(\mathrm{V} \mathrm{ps}), \\
\mu=4.0 \mu \mathrm{m}^{2} /(\mathrm{V} \mathrm{ps}) .
\end{gathered}
$$

2. Variable $\mu$ depending on the doping $n_{d}$ :

$$
\mu= \begin{cases}0.75 \mu \mathrm{m}^{2} /(\mathrm{V} \mathrm{ps}), & \text { in the } n^{+} \text {region, } \\ 4.0 \mu \mathrm{m}^{2} /(\mathrm{V} \mathrm{ps}), & \text { in the } n \text { region. }\end{cases}
$$

3. Variable $\mu$ depending on the electric field $E$, used in drift-diffusion simulations to model saturation:

$$
\mu(E)=2 \mu_{0} /\left[1+\sqrt{1+4\left(\mu_{0}|E| / v_{d}\right)^{2}}\right],
$$

where

$$
\mu_{0}=4.0 \mu \mathrm{m}^{2} /(\mathrm{V} \mathrm{ps}), \quad v_{d}=2.0 \mu \mathrm{m} / \mathrm{ps} .
$$

$v_{d}$ here is taken to be the maximum of the velocity in the kinetic run with $\nu$ bias $=1.0$ and $\mu=4$.

More specific conditions for various models are listed below. 
1. For the kinetic model (8.1):

- The velocity space is artificially cut at

$$
-a \leq u \leq a
$$

where we monitor to ensure that $f(x, u, t)$ is always very small at the boundary $u= \pm a$ for the final steady state results. We learned that it is more than enough in all our runs to use $a=3.5$. Larger values of $a$ are also used to verify that the results do not change in the pictures.

- At $x=0$, take

$$
f(0, u, t)=n_{d}(0) M(u)
$$

if $u \geq 0$, and no boundary condition (extrapolation of the numerical solution from inside the domain to the boundary) if $u<0$. Also take $\phi(0, t)=0$.

- At $x=0.8$, take

$$
f(0.8, u, t)=n_{d}(0.8) M(u)
$$
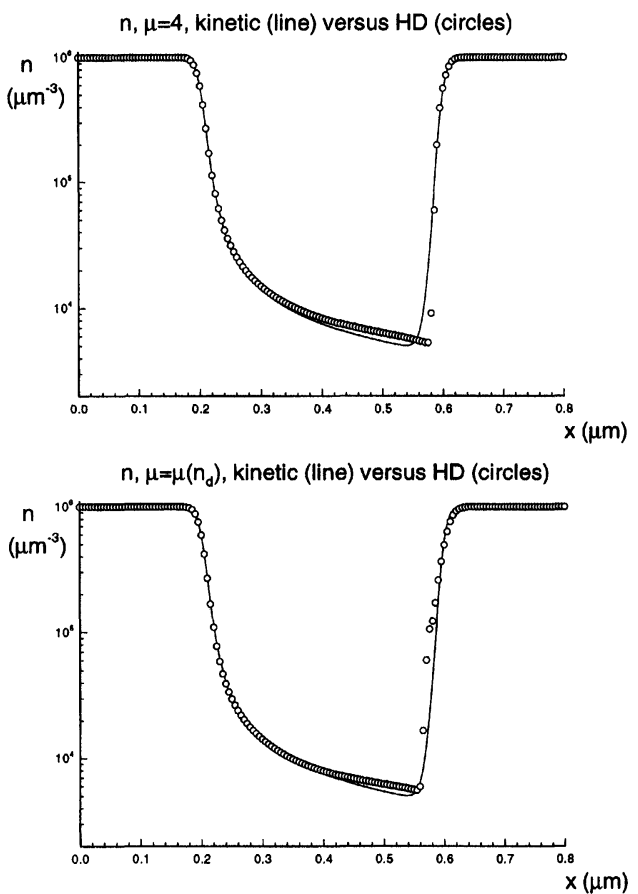

if $u \leq 0$, and no boundary condition (extrapolation of the numerical solution from inside the domain to the boundary) if $u>0$. Also take $\phi(0, t)=v$ bias.

- At $u=-a$ and $u=a$, take no boundary condition (extrapolation of the numerical solution from inside the domain to the boundary).

2. For the hydrodynamic (HD) model (1.2)-(1.4):

- At $x=0$, take

$$
n(0, t)=n_{d}(0), T(0, t)=T_{0}, \phi(0, t)=0,
$$

with other quantities extrapolated from inside the computational domain to the boundary.

- At $x=0.8$, take

$$
\begin{gathered}
n(0.8, t)=n_{d}(0.8), T(0.8, t)=T_{0}, \\
\phi(0.8, t)=v \text { bias }
\end{gathered}
$$

with other quantities extrapolated from inside the computational domain to the boundary.
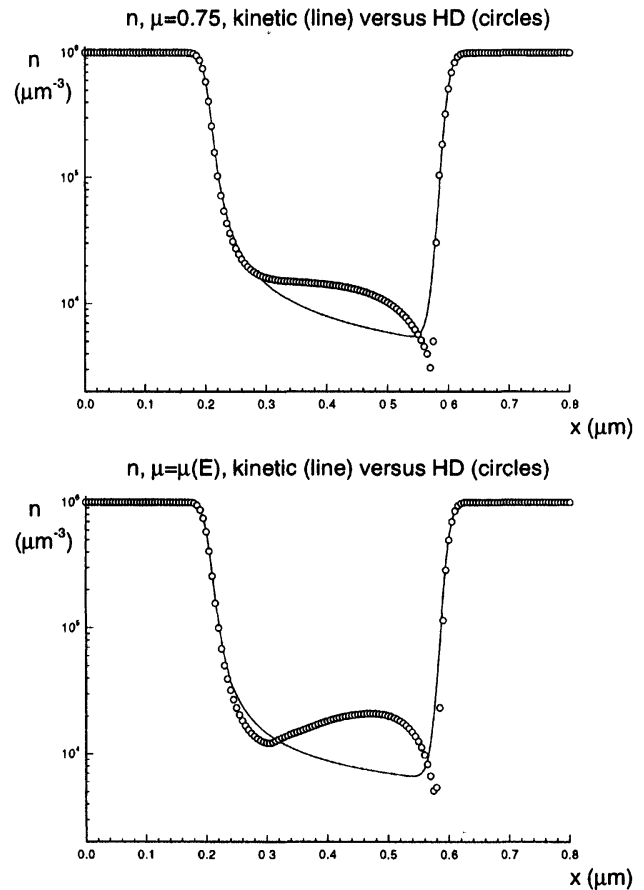

FIGURE 3 Hydrodynamic (HD) results (circles) versus kinetic simulation results (solid line), $v$ bias $=1$ volt. The concentration $n$ in $\mu \mathrm{m}^{-3}$. Top left: $\mu=4$; top right: $\mu=0.75$; bottom left: $\mu=\mu\left(n_{d}\right)$ as given by $(8.10)$; bottom right: $\mu=\mu(E)$ as given by $(8.11)$. 
We compare the simulation results of the hydrodynamic (HD) model, with various assumptions on $\mu$ as given in (8.8), (8.9), (8.10) and (8.11), with the kinetic simulation results obtained with the same mobility assumptions. Figure 3 shows the results of concentration $n$. We can see that the results between the two models are very similar for $\mu=4$ and $\mu=\mu\left(n_{d}\right)$ as given by $(8.10)$, but are quite different for $\mu=0.75$ and $\mu=\mu(E)$. Alternative simulations were carried out in [19].

\section{SIMULATIONS OF THE QHD MODEL}

One of the encouraging features of the QHD model is the recovery of two fundamental properties of quantum transport as observed in quantum structures such as the resonant tunneling diode. These are negative differential resistance and hysteresis. Below, we indicate in Figure 4 the current-voltage curves reproduced from [14].

To exhibit hysteresis, we simulate a GaAs resonant tunneling diode with double $\mathrm{Al}_{0.3} \mathrm{Ga}_{0.7} \mathrm{As}$ barriers (the barrier height $\mathcal{B}=0.209 \mathrm{eV}$ ). The doping density $n_{d}=10^{18} \mathrm{~cm}^{-3}$ in the $n^{+}$source and drain, and $n_{d}=5 \times 10^{15} \mathrm{~cm}^{-3}$ in the $n$ channel. The channel is $250 \AA$ long, the barriers are $50 \AA$ wide, and the well between the barriers is $50 \AA$ wide. The device has $50 \AA$ spacers between the barriers

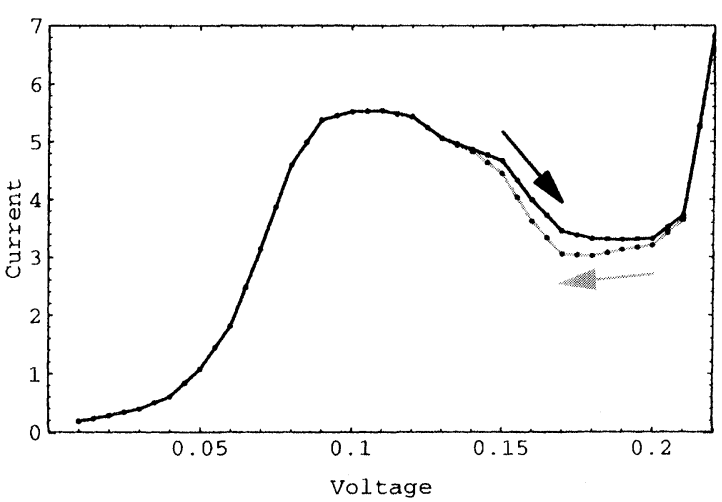

FIGURE 4 Current-voltage curve exhibiting negative differential resistance and hysteresis. and the contacts (source and drain) to enhance negative differential resistance.

The current-voltage curve for the resonant tunneling diode is plotted in Figure 4 for $\Delta V$ increasing from 0 volts to 0.22 volts (upper curve) and decreasing from 0.22 volts to 0 volts (lower curve). Note that hysteresis occurs predominantly in the region of negative differential resistance. The physical mechanism for hysteresis is that electrons "see" a different potential energy due to different accumulated electron charges in the diode when the applied voltage is decreasing than when the applied voltage is increasing.

\section{Acknowledgements}

The author is supported by the National Science Foundation under grant DMS-9704458.

\section{References}

[1] Ancona, M. G. and Iafrate, G. J. (1989). Quantum correction to the equation of state of an electron gas in a semiconductor. Physical Review, B39, 9536-9540.

[2] Ancona, M. G. and Tierston, H. F. (1987). Macroscopic physics of the silicon inversion layer. Physical Review, B35, 7959-7965.

[3] Alsunaidi, M. A. and El-Ghazaly, S. M. (1994). High frequency time domain modeling of GaAs FET's using (the) hydrodynamic model coupled with Maxwell's equations. IEEE Symp., San Diego, pp. 397-400.

[4] Alsunaidi, M. A., Imtiaz, S. S. and El-Ghazaly, S. M. (1996). Electromagnetic wave effects on microwave transistors using a full-wave time-domain model. IEEE Trans. Microwave Theory and Tech., 44, 799-808.

[5] Baccarani, G. and Wordeman, M. R. (1985). An investigation of steady-state velocity overshoot effects in $\mathrm{Si}$ and GaAs devices. Solid State Electronics, 28, 404-416.

[6] Barenger, H. U. and Wilkins, J. W. (1987). Ballistic structure in the electron distribution function of small semiconducting structures: General features and specific trends. Phys. Rev. B, 36, 1487-1502.

[7] Bløtekjær, K. (1970). Transport equations for electrons in two-valley semiconductors. IEEE Trans. Electron Devices, $17,38-47$.

[8] Cercignani, C., Gamba, I. M., Jerome, J. W. and Shu, C.-W. (2000) Device benchmark comparisons via kinetic, hydrodynamic, and high-field models. Computer Meth. Appl. Mech. Eng., 181, 381-392.

[9] Cercignani, C., Gamba, I. M., Jerome, J. W. and Shu, C.-W., A domain decomposition model for semiconductors. Poster Session, MAFPD-V, Mauii, July, 1998.

[10] Chen, G.-Q., The compensated compactness method and the system of isentropic gas dynamics. MSRI Preprint 00527-91, Berkeley, 1990. 
[11] Chen, G.-Q., Jerome, J. W., Shu, C.-W. and Wang, D. (1998). Two carrier semiconductor device models with geometric structure and symmetry properties. In: Jerome, J. (Ed.), Modelling and Computation for Applications in Mathematics, Science, and Engineering, Clarendon Press, Oxford, pp. 103-140.

[12] Chen, G.-Q., Jerome, J. W. and Wang, D. (2000) Compressible Euler-Maxwell equations. J. Transport Th. Statistical Phys., 29.

[13] Chen, Z., Cockburn, B., Jerome, J. W. and Shu, C.-W. (1995). Mixed-RKGD finite element methods for the 2-D hydrodynamic model for semiconductor device simulation. VLSI DESIGN, 3, 145-158.

[14] Chen, Z., Cockburn, B., Gardner, C., Jerome, J. W. (1995). Quantum hydrodynamic simulation of hysteresis in the resonant tunneling diode. J. Comp. Phys., 117, 274-280.

[15] Cockburn, B., Hou, S. and Shu, C.-W. (1990). TVB Runge-Kutta local projection discontinuous Galerkin finite element method for conservation laws IV: the multidimensional case. Math. Comp., 54, 545-581.

[16] Ding, X., Chen, G.-Q. and Luo, P. (1987). Convergence of the Lax-Friedrichs scheme for isentropic gas dynamics (I)-(II). Acta Math. Scientia, 7, 467-480; 8, 61-94, 1988.

[17] Fatemi, E., Gardner, C., Jerome, J., Osher, S. and Rose, D. (1991). Simulation of a steady-state electron shock wave in a submicron semiconductor device using high order upwind methods. In: Hess, K., Leburton, J. P. and Ravaioli, U. (Eds.), Computational Electronics. Kluwer Academic Publishers, Boston Dordrect, pp. 27-32.

[18] Fatemi, E., Jerome, J. and Osher, S. (1991). Solution of the hydrodynamic device model using high-order nonoscillatory shock capturing algorithms. IEEE Trans. Computer-Aided Design of Integrated Circuits and Systems, CAD-10, 232-244.

[19] Fatemi, E. and Odeh, F. (1993). Upwind finite difference solution of the Boltzmann equation applied to electron transport in semiconductor devices. J. Comput. Phys., 108, $209-217$.

[20] Gardner, C. L. (1994). The quantum hydrodynamic model for semiconductor devices. SIAM J. Appl. Math., 54, $409-427$.

[21] Grubin, H. L. and Kreskovsky, J. P. (1989). Quantum moment balance equations and resonant tunnelling structures. Solid-State Electronics, 32, 1071-1075.

[22] Jerome, J. W., Analysis of Charge Transport: A Mathematical Study of Semiconductor Devices, Springer-Verlag, Heidelberg, 1996.

[23] Jerome, J. W. and Shu, C.-W., Energy models for onecarrier transport in semiconductor devices. In: IMA Volumes in Mathematics and Its Applications, Vol. 59 (Eds. Coughran, W., Cole, J., Lloyd, P. and White, J.), pp. 185-207, Springer, 1994.

[24] Jerome, J. W. and Shu, C.-W. (1995). Transport effects and characteristic modes in the modeling and simulation of submicron devices. IEEE Trans. on Computer-Aided Design, 14, 917-923

[25] Jerome, J. W. and Shu, C.-W. (1995). The response of the hydrodynamic model to heat conduction, mobility, and relaxation expressions. VLSI Design, 3, 131-143.

[26] Marcati, P. and Natalini, R. (1995). Weak solutions to a hydrodynamic model for semiconductors \& relaxation to the drift-diffusion equation. Arch. Rat. Mech. Anal., 129, $129-145$.

[27] Rudan, M., Odeh, F. and White, J. (1987). Numerical solution of the hydrodynamic model for a one-dimensional semiconductor. COMPEL (The International Journal for Computation and Mathematics in Electronic Engineering), 6, 151-170.

[28] Selberherr, S., Analysis and Simulation of Semiconductor Devices, Springer-Verlag, Wien-New York, 1984.

[29] Shu, C.-W. and Osher, S. J. (1988). Efficient implementation of essentially non-oscillatory shock capturing schemes. J. Comp. Phys., 77, 439-471.

[30] Shu, C.-W. and Osher, S. J. (1989). Efficient implementation of essentially non-oscillatory shock capturing schemes, II. J. Comp. Phys., 83, 32-78.

[31] Stettler, M. A., Alam, M. A. and Lundstrom, M. S. (1993). A critical examination of the assumptions underlying macroscopic transport equations for silicon devices. IEEE Trans. Electron Devices, 40, 733-740.

[32] Tartar, L., Compensated compactness and applications to partial differential equations, Research Notes in Mathematics, Nonlinear Analysis and Mechanics, Vol. 4 (Ed. Knops, R. J.). Pitman Press, 1979.

[33] Zhang, B. and Jerome, J. W. (1996). On a steady-state quantum hydrodynamic model for semiconductors. Nonlinear Anal., 26, 845-856.

\section{Author Biography}

Joseph W. Jerome received the Ph. D. degree in Mathematics from Purdue University in 1966 . He was visiting Assistant Professor at the Mathematics Research Center, University of Wisconsin, during 1966-68, and was Assistant Professor at Case Western Reserve University during 1968-70. He joined Northwestern University in 1970, where he has been Professor of Mathematics and Applied Mathematics since 1976. He has held sabbatical positions at Oxford University, England, 197475, University of Texas, Austin, 1978-79, and Bell Laboratories, Murray Hill, 1982-83. He was visiting scholar at the University of Chicago in 1985. He received the Distinguished Alumnus Award from Purdue University's School of Science in 1996. His research interests include applied analysis, approximation theory, numerical analysis, computational electronics, and ion transport in biology. The most recent of his three books, Analysis of Charge Transport: A Mathematical Study of Semiconductor Devices, was published by Springer in 1996. 

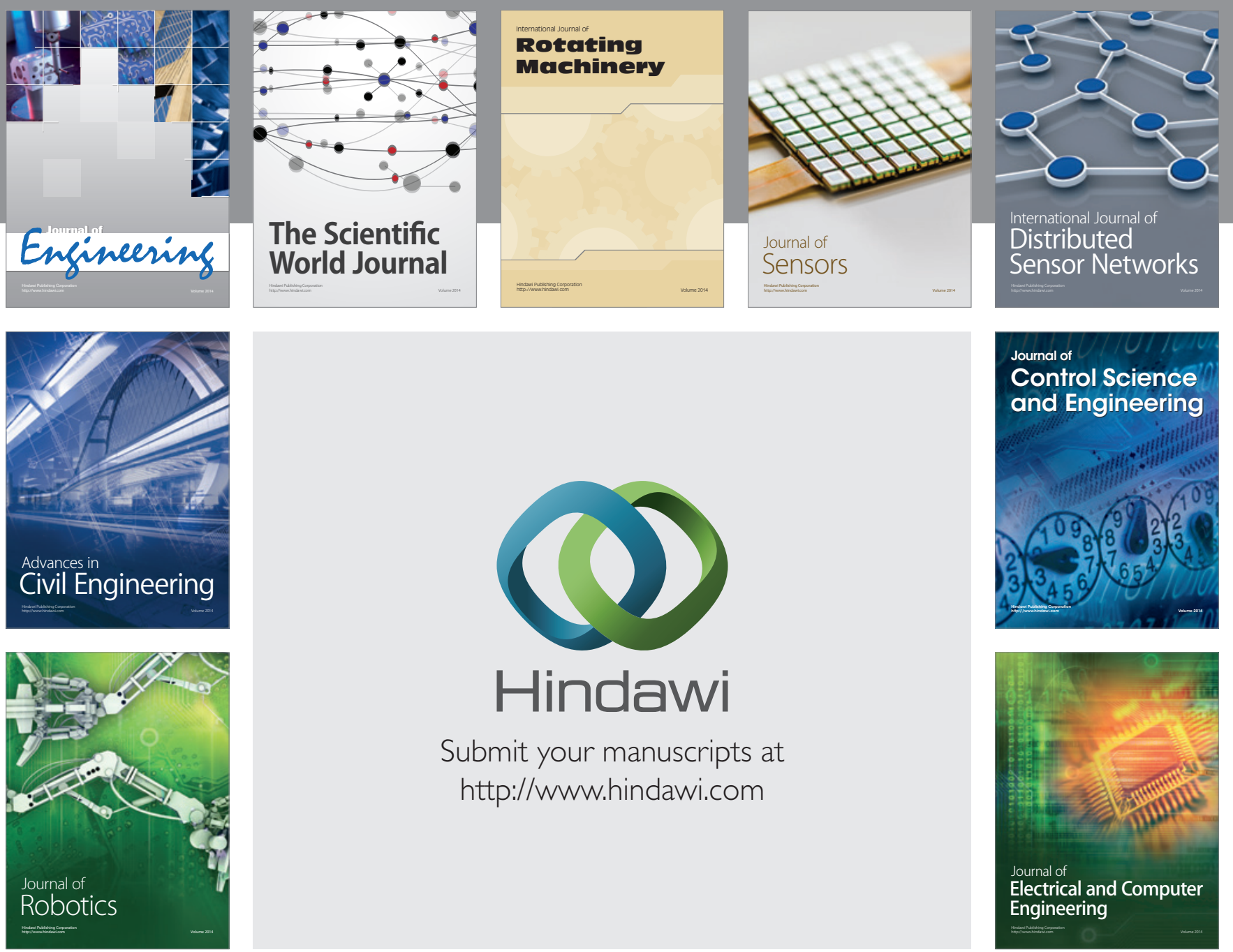

Submit your manuscripts at

http://www.hindawi.com
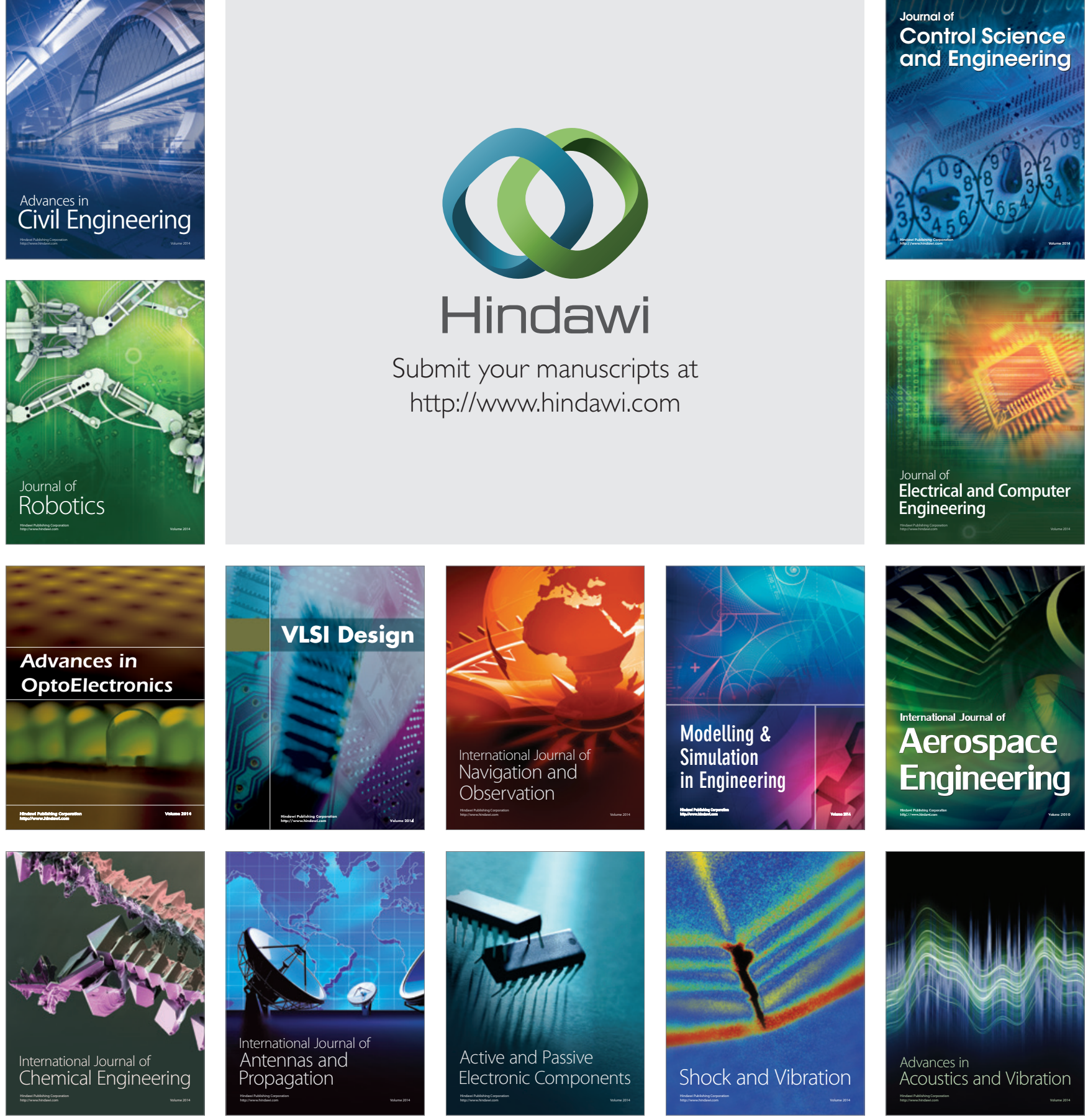